Case Report: Open Access

\title{
Successful Treatment of a Primary Cervical Diffuse Large B-cell Lymphoma with Rituximab-CHOP Immunochemotherapy
}

\author{
Díaz De-La-Noval B*, Hernández Gutiérrez A, Zapardiel I, De-Santiago García J and \\ Diestro Tejeda
}

Gynecology and Obstetrics Department, Gynecology Oncology Unit, La Paz University Hospital, Madrid, Spain

*Corresponding author: Díaz De-La-Noval B, Gynecology and Obstetrics Department, Gynecology Oncology Unit, Hospital Universitario La Paz, Madrid-Spain, Paseo de la Castellana 261, 28046-Madrid, Spain, Tel: +34616306566, E-mail: begodelanoval@gmail.com

\begin{abstract}
Introduction: Primary Large B-cell lymphoma of the cervix is a very rare disease. Because of the rarity of this tumor, it can lead to mistakes or delays in the diagnosis and proper therapy, and eventually becoming diagnosed in locally advanced stages.

Objective: The aim is to provide an updated review in the issue and report a recent case in our institution.

Case report: We describe a 46-year-old healthy woman with primary extranodal non-Hodgkin's diffuse large B-cell lymphoma (DLBCL) of the uterine cervix Ann Arbor stage IIEA IPI 1. The patient presented an acute renal failure and severe ureterohydronephrosis due to a large cervical mass. She was successfully treated with 6 courses of multidrug therapy based on Rituximab, Cyclophosphamide, Doxorubicin, Vincristine and Prednisone (R-CHOP) with no major side effects.

Discussion: Symptoms are usually nonspecific and the diagnosis is difficult to reach. DLBCL is the most common histological type of extranodal non-Hodgkin's lymphoma. Therapy is still under discussion but chemotherapy with $\mathrm{R}-\mathrm{CHOP}$ or $\mathrm{CHOP}$-like regimens are suitable with a better prognosis and high overall survival.

To conclude, in the case of a young woman with a large well defined uterine cervical mass, bilateral ureteral entrapment and an uncertain Pap or biopsy tests, the presence of a lymphoma should be considered among other rare tumors. Biopsy and immunophenotypical evaluation are essential for diagnosis of unusual tumors. We should not establish a treatment till have a correct diagnosis.
\end{abstract}

\section{Keywords}

Non-Hodgkin's lymphoma, Diffuse large B-cell lymphoma, Extranodal lymphoma, Cervical carcinoma

\section{Introduction}

The incidence of Non-Hodgkin's Lymphoma (NHL) has been increasing in recent decades, the prevalence of extranodal-NHL is about 30 to $50 \%$ of all NHL patients. Primary Large B-cell extranodal lymphoma of the cervix is a very rare disease, $0.008 \%$ of all cervical tumors. The Diffuse large B-cell lymphoma (DLBCL) is the most common extranodal lymphoid neoplasm in around $25 \%$ of all NHL [1]. DLBCL has an aggressive course with a rapidly growing mass. The gastrointestinal tract and the skin are the most common estranodal sites, but almost any organ can be affected. Primary lymphomas of the female reproductive tract are less than $1 \%$ of extranodal-NHL and $<0.5 \%$ of gynecological cancers. Primary pelvic NHL extranodal sites include the ovary, uterus, cervix, vagina, vulva and regional lymph nodes, with the cervix being the most common. Because of the rarity of this tumor, the diagnosis is difficult and therapy delayed, so is often detected at an advanced stage [2].

\section{Case Report}

In February 2015, a 46-year-old woman developed an obstructive acute renal failure and severe ureterohydronephrosis. Since previous year, the patient suffered from recurrent urinary infections, hematuria, and pain on the left flank, suspecting urolithiasis. She did not refer pelvic pain but little persistent vaginal spotting and dyspareunia appeared months before. No relevant personal history except smoking and autoimmune thyroiditis undergoing a total thyroidectomy for papillary thyroid carcinoma. An abdominal ultrasound showed a small right kidney with signs of residual repeated pyelonephritis and moderate left hydronephrosis with no lithiasis. An urological scanner (Uro-CT) revealed a cervical mass. Serum creatinine level was $5.63 \mathrm{mg} / \mathrm{dl}$ and pyelogram displayed a severe bilateral ureterohydronephrosis with a distal stop. Considering a secondary obstructive acute pre-renal failure and severe bilateral hydroureters, the patient required nephrostomies (Figure 1).

The gynecological evaluation reported a $6 \mathrm{~cm}$ bulky endophytic cervical mass which extended to the upper third of the vagina and both parametrium, left almost to the pelvic wall; there was no inguinal lymphadenopathy, uterus and adnexae appeared normal. Positron Emission Tomography-Computed Tomography (PET-CT) showed additional left iliac lymph nodes without retroperitoneal invasion (Figure 2). Tumor markers altered were SCC $2.1 \mathrm{ng} /$ $\mathrm{ml}$ and $\beta 2-\mu g l o b u l i n ~ 5.5 \mathrm{mg} / \mathrm{dl}$. Pap test and superficial cervical biopsy were negative, but suspicion was a clinical locally-advanced cervical carcinoma International Federation of Gynecology and Obstetrics (FIGO) stage IIIB. We decided to perform a gynecological 


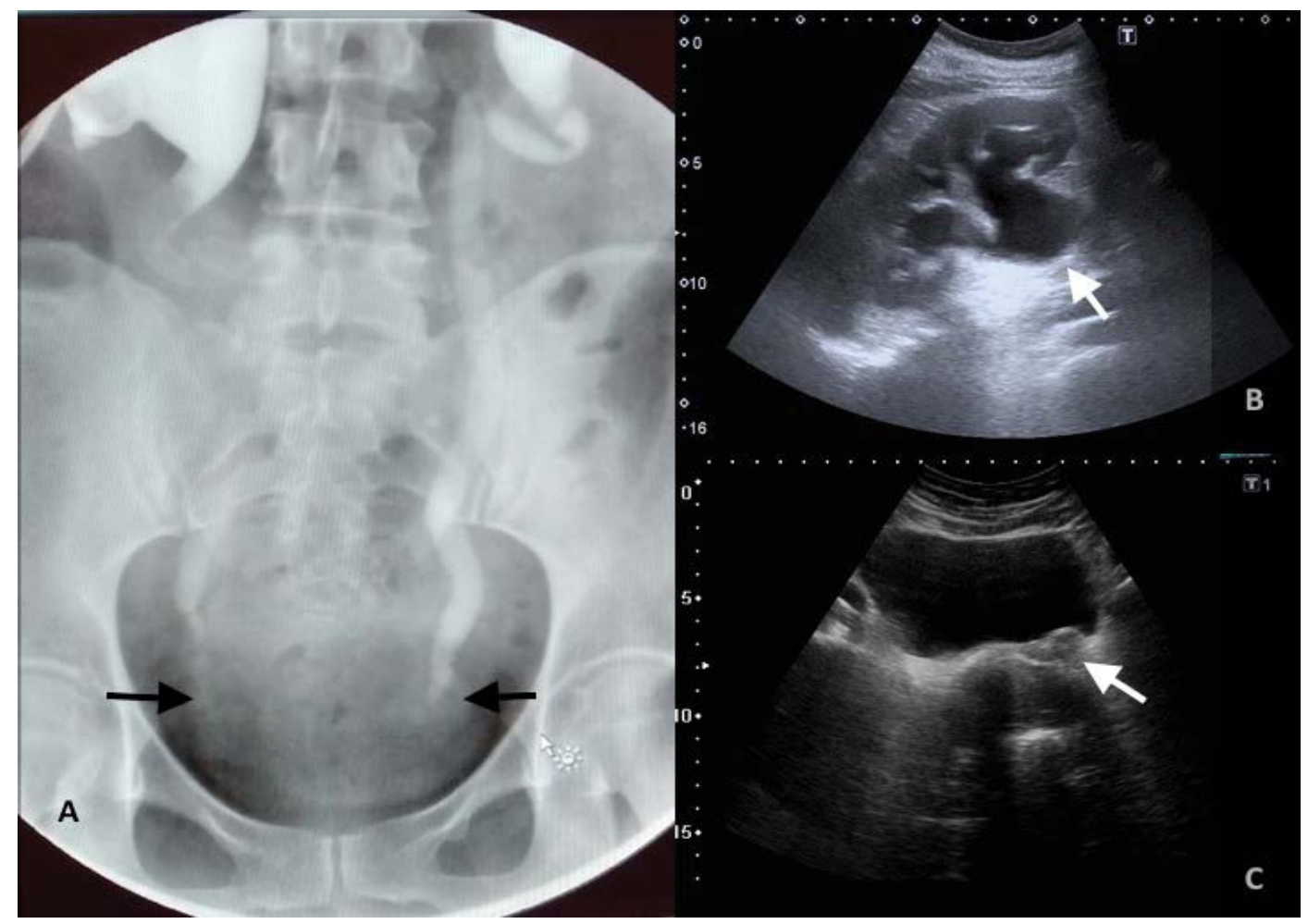

Figure 1: (A) Pyelogram: severe bilateral hydronephrosis with a distal stop in both sides; $(B, C)$ Abdominal echography; (B) severe dilatation of the renal pelvis with cortical thinning in left kidney; $(C)$ and a high vascularised $6 \mathrm{~cm}$ cervical mass that expands to the posterior wall of the bladder trigone and traps both ureters. Uterus and adnexae appeared normal.

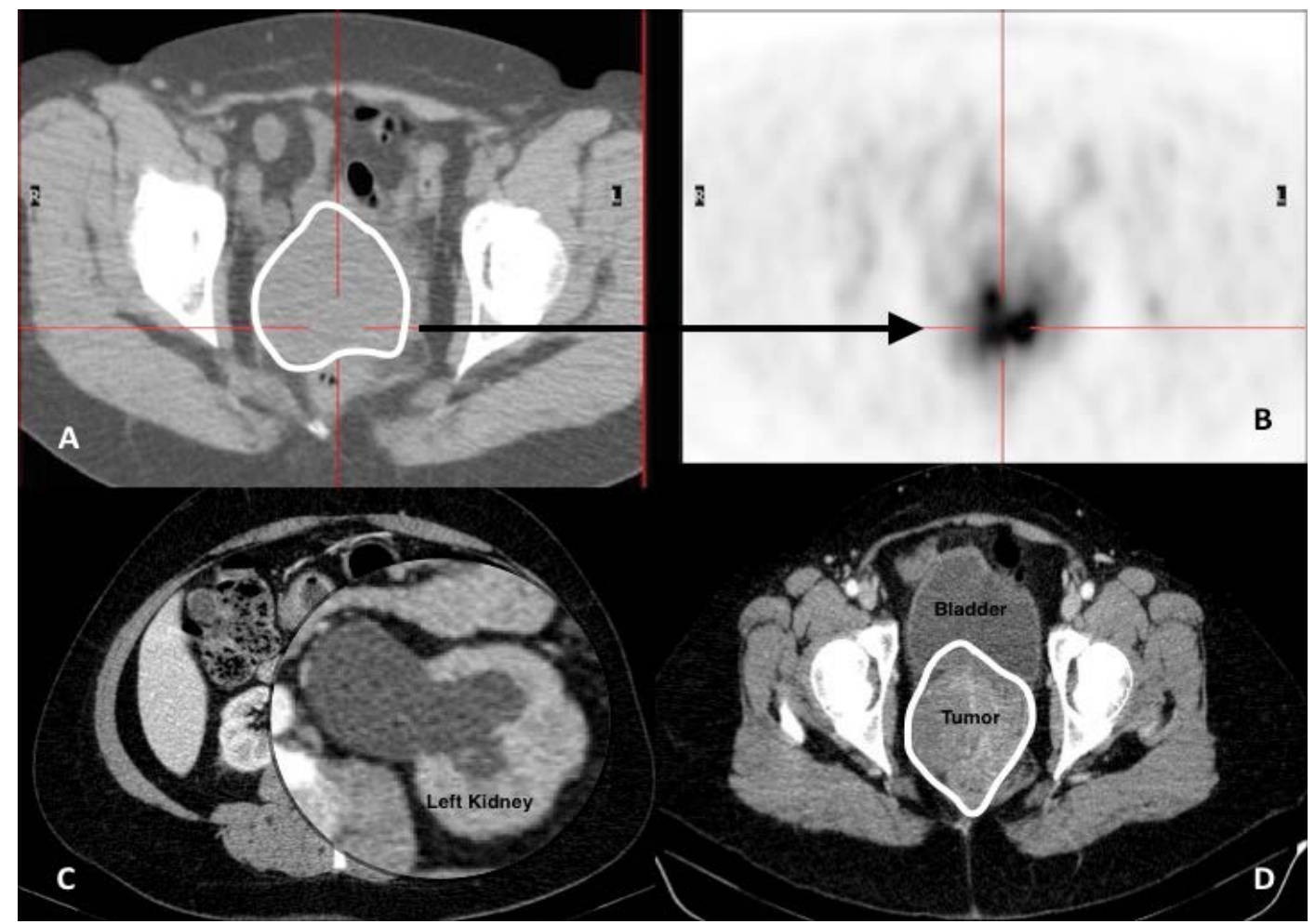

Figure 2: $(A, B)$ PET described an intense well defined FDG avid mass in the uterine cervix.; $(C, D) C T$ at diagnosis: Bulky tumor mass involving the whole cervix and reaching the posterior wall of the bladder with bilateral ureteral entrapment and severe hydronephrosis, mostly on the left side.

examination under anesthesia with a conization and deep biopsies to ensure the diagnosis.

Histological examination showed a diffuse infiltration of medium to large sized mononuclear cells with atypical fusiform ones around vascular structures but not infiltrating. Immunohistochemical staining was CD20, CD45, BCL6 and CD30 positive, 50\% Ki67, with CK-AE1/AE3, CD15, AML, CD68, BCL2, CD10, MUM1, CD5 and C-MYC negative. Hans's Algorithm suggested a Germinal Center
B-cell Lymphoma (GCB). Further staging has not revealed any other localization of disease.

The final diagnosis was a primary high-grade extranodal DLBCL type GCB, Ann Arbor stage IIEA1. The age-adjusted International Prognostic Index (IPI) was 1.

The patient was successfully treated with 6 courses of R-CHOP chemotherapy (CT), the standard protocol for this tumor, well 
tolerated. Actually, the patient is free of disease. She maintains right ureteral catheter due to residual ureteral fibrosis after tumor disappearance, and she has lost the left kidney function.

\section{Discussion}

DLBCL is the most common extranodal NHL neoplasm (30$58 \%)$. The median age of onset described in DLBCL is 40 -yearsold, earlier than median age of NHL (60-65 years old). Follicular lymphoma is the second most common subtype of lymphoma and represents about $20 \%$ to $25 \%$ of all cases of NHL, others are B-cell small lymphocytic lymphoma, mantle-cell lymphoma, follicular centre-cell lymphomas, and marginal zone B-cell lymphoma (it includes MALT lymphoma) with a rate of $17 \%, 12.8 \%, 4.3 \%$, and $2.1 \%$, respectively [3].

Symptoms are usually non-specific and include abnormal vaginal bleeding as the most common $(70 \%)$ but it often presents with bilateral ureterohydronephrosis, other symptoms are perineal discomfort $(40 \%)$, persistent vaginal discharge $(20 \%)$, abdominal pain or asymptomatic. B-symptoms of NHL were rarely seen [4].

The diagnosis may be difficult to reach, and usually requires histological review and immunohistochemical evaluation. Pap is typically negative so colposcopy and a deep biopsy are essential to establish the diagnosis. That is because these tumors arise from the cervical stroma, and the overlying squamous epithelial is preserved, at least initially [2].

Computed Tomography (CT) is commonly the study of choice for detection and staging of NHL. Staging also includes a bone marrow biopsy and PET-TC [3].

Immunohistochemistry is necessary to determine the histological type of lymphoma. Hans's Algorithm uses the expression of CD20, CD10, BCL6 y MUM1. In our case, CD20 positive, CD10 negative, BCL6 positive and MUM-1 negative suggested a DLBCL type GCB $[4,5]$. Lactate dehydrogenase (LDH) is a good prognostic marker of Diffuse Large B-cell Lymphoma, but in our case LDH was not requested because a lymphoma was not first suspected, subsequent tests during and after treatment had normal levels.

The differential diagnosis is usually done with small-cell neuroendocrine carcinoma, undifferentiated cervical carcinoma and adenocarcinoma of the cervix [1].

Staging is performed according to the Ann Arbor System, with stages I and II being most common. Stage I with an involvement of a single lymph node area or location. Stage II with two or more lymph node areas or extranodal territory affected on the same side of the diaphragm. Extranodal NHL is represented with an "E". Adding an "A" to the stage denotes the absence of constitutional symptoms. The presence of those symptoms is denoted by adding a " $\mathrm{B}$ " to the stage.

At diagnosis, 30\% cervical NHL are in an early stage (I or II) and $30-40 \%$ have extranodal involvement. Between $20-30 \%$ of patients have bone marrow involvement, and 5-10\% have metastasis in the central nervous system when relapse or progression [6].

The IPI is considered the most reliable and reproducible prognostic model to quantify the evolution of a NHL disease. IPI combines five clinical parameters easily measured: tumor stage, serum LDH level, number of extranodal disease sites, age and performance status. One point is assigned for each of the following risk factors: age greater than 60 years, stage III or IV disease, elevated serum LDH, performance status of 2, 3, or 4, and more than 1 extranodal site. IPI is classified into four risk groups: low (0-1 points, $73 \%$ 5-year OverallSurvical (OS)), medium-low (2 points, $51 \% 5$-year OS), intermediatehigh ( 3 points, $43 \% 5$-year OS) and high (4-5 points, $26 \% 5$-year OS). Low-risk group has a predicted 4-year progression-free survival and OS of $94 \%$ [6], our patient had a low-risk IPI value.

The DLBCL has an aggressive course but potentially curable. Therapy is still under discussion. Actually, R-CHOP is one of the most used CT regimen [7]. with a completed 6 cycles of R-CHOP poly-CT, $70 \%$ to $80 \%$ of complete remission is obtained in Ann Arbor stages I and II. There are other more intensive regimens as R-ACVBP (rituximab plus doxorubicin, cyclophosphamide, vindesine, bleomycin, and prednisone) or R-EPOCH (rituximab plus etoposide, prednisolone, oncovin, cyclophosphamide and hydroxydaunorubicin), but worse tolerated and more adverse effects. In our patient, due to renal failure, these intensive CT schemes were not advised; so, intensive regimens are preferred for poor-prognosis, more advanced disease or in aggressive non-Hodgkin Lymphoma.

Radiotherapy is considered for residual lesions, large tumors (> $10 \mathrm{~cm}$ ) or incomplete response to primary CT. Radical surgery should be avoided [1]. The OS rate for patients with lymphoma of the cervix, vagina, and endometrium is $89 \%$, with a disease free survival rate of $70 \%$. The 5-year OS of patients with GCB lymphoma is higher [4].

In conclusion, although Primary Large Bcell extranodal lymphoma of the cervix is a rare disease and the squamous cell carcinoma is the most common type of malignancy; in case of a young woman with a quickly developed Bulky uterine cervical mass, bilateral hydronephrosis and an uncertain Pap or biopsy tests, the presence of a lymphoma should be considered among other rare tumors. Given the aggressiveness of the tumor, a soon diagnosis is necessary. Histological and immunophenotypical evaluation are essential for diagnosis of unusual tumors. We should not establish any treatment till having the correct diagnosis. First line therapy of a lymphoma is CT with good results.

\section{Ethical Statement}

The authors declare that they have no competing interests. Written consent from the patient.

\section{References}

1. Mandato VD, Palermo R, Falbo A, Capodanno I, Capodanno F, et al. (2014) Primary diffuse large B-cell lymphoma of the uterus: case report and review. Anticancer Res 34: 4377-4390.

2. Bellevicine C, Zabatta A, Malapelle U, Vetrani A, Troncone G (2014) Diffuse large B-cell extranodal lymphoma of the uterine cervix: an incidental pap smear finding with histological and immunohistochemical correlation. Diagn Cytopathol 42: 644-646.

3. Novotny S, Ellis T, Stephens J (2011) Primary B-cell lymphoma of the cervix presenting with bilateral hydronephrosis. Obstet Gynecol 117: 444-446.

4. Ahmad AK, Hui P, Litkouhi B, Azodi M, Rutherford T, et al. (2014) Institutional review of primary non-hodgkin lymphoma of the female genital tract: a 33year experience. Int J Gynecol Cancer 24: 1250-1255.

5. Campo E, Swerdlow SH, Harris NL, Pileri S, Stein H, et al. (2011) The 2008 WHO classification of lymphoid neoplasms and beyond: evolving concepts and practical applications. Blood 117: 5019-5032.

6. Cao XX, Li J, Zhang W, Duan MH, Shen T, et al. (2014) Patients with primary diffuse large B-cell lymphoma of female genital tract have high risk of central nervous system relapse. Ann Hematol 93: 1001-1005.

7. Egyed M, Kollár B, Prievara FT, Viski A, Bajzik G, et al. (2007) Successful treatment of a primary uterine B-cell lymphoma with rituximab-CHOP immunochemotherapy. Haematologica 92: e26-27. 